PROCEEDINGS OF THE

AMERICAN MATHEMATICAL SOCIETY

Volume 131, Number 11, Pages 3499-3505

S 0002-9939(03)06906-5

Article electronically published on April 1, 2003

\title{
LOCAL BIFURCATION FROM THE SECOND EIGENVALUE OF THE LAPLACIAN IN A SQUARE
}

\author{
MANUEL DEL PINO, JORGE GARCÍA-MELIÁN, AND MONICA MUSSO
}

(Communicated by David S. Tartakoff)

\begin{abstract}
In this work we study local bifurcation from the branch of trivial solutions for a class of semilinear elliptic equations, at the second eigenvalue $\lambda_{2}$ of a square. We find that the bifurcation set can be locally described as the union of exactly four bifurcation branches of nontrivial solutions which cross the bifurcation point $\left(\lambda_{2}, 0\right)$. We also compute the Morse index of the solutions in the four branches.
\end{abstract}

\section{INTRODUCTION}

The question of local bifurcation from the branch of trivial solutions $(\lambda, 0)$ in an equation of the form

$$
\left\{\begin{array}{cc}
\Delta u+\lambda u+f(\lambda, x, u)=0 & \text { in } \quad \Omega, \\
u=0 & \text { on } \partial \Omega,
\end{array}\right.
$$

where $\lambda \in \mathbb{R}, \Omega \subset \mathbb{R}^{N}$ is a bounded domain and $f(\lambda, x, u)=o(u)$ as $u \rightarrow 0$, has been widely treated in the literature. We say that bifurcation takes place at $\lambda=\bar{\lambda}$ if every neighborhood of $(\bar{\lambda}, 0)$ in $\mathbb{R} \times C(\bar{\Omega})$ contains a nontrivial solution $(\lambda, u)$ of (1.1). Under mild regularity assumptions on $f$, it is easy to check that the only possible values of $\bar{\lambda}$ for which bifurcation is possible are the eigenvalues $\lambda_{1}<\lambda_{2}<\cdots$ of the problem

$$
\left\{\begin{array}{ccc}
\Delta \phi+\lambda \phi=0 & \text { in } & \Omega \\
\phi=0 & \text { on } & \partial \Omega
\end{array}\right.
$$

On the other hand, since problem (1.1) has a potential structure, it follows from the results in $[2,4,[5,6]$, that the reciprocal also holds true: bifurcation occurs at $\lambda=\lambda_{k}$ for any $k \geq 1$.

A natural problem is that of finding the structure of the bifurcating set at a given eigenvalue $\lambda_{k}$, namely that of the set of nontrivial solutions $(\lambda, u)$ of (1.1) in a neighborhood of $\left(\lambda_{k}, 0\right)$. A well-known result by Crandall and Rabinowitz [3] provides an accurate description in the case when the eigenvalue $\lambda_{k}$ is simple, say with eigenspace spanned by a function $\phi_{k}$ : the bifurcation set is (locally) a $C^{1}$ curve which can be parametrized as $s \mapsto(\lambda(s), u(s))$ in $\mathbb{R} \times C(\bar{\Omega})$ with

$$
(\lambda(0), u(0))=\left(\lambda_{k}, 0\right), \quad u^{\prime}(0)=\phi_{k} .
$$

Received by the editors June 2, 2002.

2000 Mathematics Subject Classification. Primary 35B32, 35J25.

Key words and phrases. Local bifurcation, multiple branches, double eigenvalue. 
Concerning eigenvalues of higher multiplicity, it follows from the results in [6] and the potential structure of the problem that the following alternative holds true at any eigenvalue $\lambda_{k}$ : (i) For $\lambda=\lambda_{k}$ the trivial solution $u=0$ of (1.1) is not isolated; or (ii) for any $\lambda$ in a one-sided neighborhood of $\lambda_{k}$, there are at least two nontrivial solutions, or (iii) for any $\lambda$ in a neighborhood of $\lambda_{k}$, at least one nontrivial solution exists. One can roughly portray this situation as that associated to the presence of a bifurcating curve transversally crossing the point $\left(\lambda_{k}, 0\right)$. On the other hand, examples are known of potential operators where bifurcation takes place in the form of a discrete set [2].

A natural question is whether accurate descriptions parallel to that in CrandallRabinowitz theorem are still possible at eigenvalues of (1.2) with multiplicity greater than one, at least in special cases.

We restrict ourselves in what follows to a special case of problem (1.1):

$$
\left\{\begin{array}{cl}
\Delta u+\lambda u+u^{3}(1+\theta(x, u))=0 & \text { in } \Omega, \\
u=0 & \text { on } \partial \Omega,
\end{array}\right.
$$

where $\theta \in C^{1}(\bar{\Omega} \times \mathbb{R}), \theta(x, 0)=0$.

Let us first consider the case of the unit disk in $\mathbb{R}^{2}$,

$$
D=\left\{(x, y) / x^{2}+y^{2}<1\right\} .
$$

We observe that the line $x=0$ splits $D$ into two half-disks. The first eigenvalue of problem (1.2) in the half-disk corresponds precisely to the second eigenvalue $\lambda_{2}$ of $D$. From Crandall-Rabinowitz theorem, a bifurcation curve of nontrivial solutions for problem (1.3) in the half-disk stems from this eigenvalue. Let us assume for instance that $\theta \equiv 0$ in (1.3). Then, extending these solutions by odd reflection with respect to the line $x=0$, we find a curve of nontrivial solutions for (1.3) now in $\Omega=D$. Since the problem in $D$ is invariant under rotations we therefore obtain a two-dimensional manifold of nontrivial solutions stemming from $\lambda=\lambda_{2}$. Observe that $\lambda_{2}$ is a double eigenvalue of the Laplacian in $D$. The tangent space to this manifold at the point $\left(\lambda_{2}, 0\right)$ is precisely the two-dimensional associated eigenspace.

Let us now consider the case of a square,

$$
S=(0, \pi) \times(0, \pi) \subset \mathbb{R}^{2} .
$$

The eigenvalues of problem (1.2) in $\Omega=S$ are easily computed with the aid of Fourier series. One finds in particular that $\lambda_{2}=5$, with multiplicity two: the eigenspace associated is that spanned by the functions $\sin 2 x \sin y$ and $\sin x \sin 2 y$. For later reference we introduce the normalized eigenfunction

$$
\phi_{\alpha}(x, y)=\cos \alpha \sin x \sin 2 y+\sin \alpha \sin y \sin 2 x,
$$

$\alpha \in[0,2 \pi)$, which is a parametric representation of all eigenfunctions $\phi$ with $|\phi|_{L^{2}}^{2}=$ $\pi / 2$.

The nodal line of $\phi_{\alpha}$ divides $S$ into two similar domains: in particular, rectangles for $\alpha=0, \pi / 4$, and triangles for $\alpha=\pi / 4$ and $\alpha=3 \pi / 4$, whose first eigenvalue equals 5. Again for $\theta \equiv 0$, Crandall-Rabinowitz theorem and extension by odd reflection with respect to the nodal line yield respectively four bifurcation curves for $\Omega=S$. The situation we encounter here is however drastically different to that of the disk: as a consequence of our main result below, the bifurcating set is constituted exactly by the union of these four curves. This phenomenon is robust: it holds true for $\theta \not \equiv 0$ as well. 
Theorem 1.1. Let $\Omega=(0, \pi) \times(0, \pi) \subset \mathbb{R}^{2}$. Then there exist an $\varepsilon>0$ and a neighborhood $\mathcal{U}$ of $\left(\lambda_{2}, 0\right)$ in $\mathbb{R} \times C(\bar{\Omega})$ such that the set of all solutions of (1.3) in $\mathcal{U}$ can be described as the union of four $C^{1}$ curves in $\mathbb{R} \times C(\bar{\Omega})$,

$$
s \in(-\varepsilon, \varepsilon) \mapsto\left(\lambda_{i}(s), u_{i}(s)\right), \quad i=1, \ldots, 4,
$$

such that

$$
\left\{\begin{array}{l}
\lambda_{i}(s)=\lambda_{2}+\sigma_{i} s^{2}+o\left(s^{2}\right) \\
u_{i}(s)=s \phi_{\alpha_{i}}+o(s)
\end{array}\right.
$$

where $\alpha_{1}=0, \alpha_{2}=\pi / 4, \alpha_{3}=\pi / 2, \alpha_{4}=3 \pi / 4$, and

$$
\begin{aligned}
\sigma_{i} & =-\frac{9}{16}, \quad \text { for } i=1,3, \\
\sigma_{i} & =-\frac{21}{32}, \quad \text { for } i=2,4 .
\end{aligned}
$$

The solutions obtained for $i=1,3$ have Morse index 3 , while those with $i=2,4$ have Morse index 2.

We specify that by Morse index of a solution $(\lambda, u)$ of (1.3) we mean the number of negative eigenvalues $\mu$ of the linear problem

$$
\left\{\begin{array}{cl}
\Delta v+\lambda v+u^{2}\left(3(1+\theta(x, u))+u \theta_{u}(x, u)\right) v+\mu v=0 & \text { in } \quad \Omega, \\
v=0 & \text { on } \partial \Omega .
\end{array}\right.
$$

We observe that in the situation described the four bifurcation curves depart left, so that in particular for $\lambda<\lambda_{2}$ sufficiently close to $\lambda_{2}$, at least four nontrivial solutions $u$ of (1.3) exist. The situation when the nonlinearity $u^{3}$ is changed to $-u^{3}$ is analogous: in this case the bifurcation occurring at $\left(\lambda_{2}, 0\right)$ still consists of four branches $(\lambda, u)$ of the form (1.5), except that the numbers $\sigma_{i}$ in (1.6) have opposite sign, being all positive. Thus the four branches bend right $\left(\lambda_{2}, 0\right)$ and existence of four solutions with small $L^{\infty}$-norm is ensured for $\lambda>\lambda_{2}$ and close to $\lambda_{2}$. Also the Morse indices of these solutions change, reducing by one unit: for $i=1,3$ their Morse index equals 2, while for $i=2,4$ it equals 1 . We should also mention that the nonlinearity $u^{3}$ could be replaced by $u^{2 k+1}, k \geq 1$, with basically no change in the proofs below.

The described phenomenon opens questions concerning the presence of multibranch bifurcation for domains enjoying other symmetries. The square is invariant under rotations around its center exactly for the four angles $0, \frac{\pi}{4}, \frac{\pi}{2}, \frac{3 \pi}{4}$ which correspond to the four values of $\alpha$ in Theorem 1.1 at which bifurcation occurs. Thus one may wonder about the corresponding diagram of nontrivial solutions for (1.3) if $\Omega$ is, for instance, a regular polygon in $\mathbb{R}^{2}$. It would be natural to expect the presence of multiple branches stemming from a double eigenvalue in that case.

The paper is organized as follows: in Section 2 we prove the first part of Theorem 1.1, while Section 3 is devoted to the calculation of the Morse index of the solutions.

\section{Construction of the Bifurcation BRAnChes}

Let us begin with some notation: we label as $M$ the vector space of all eigenfunctions associated to $\lambda_{2}$ and denote

$$
M^{\perp}=\left\{u \in C(\bar{\Omega}): \int_{\Omega} u \phi=0, \forall \phi \in M\right\} .
$$


Alongside with $\phi_{\alpha}$ in (1.4) we introduce an orthogonal eigenfunction defined by

$$
\psi_{\alpha}(x, y)=\sin \alpha \sin x \sin 2 y-\cos \alpha \sin y \sin 2 x
$$

( $\psi_{\alpha}$ is nothing but a rotation in $\pi / 2$ of $\phi_{\alpha}$ ). Notice that the derivative of $\phi_{\alpha}$ with respect to $\alpha$ coincides with $-\psi_{\alpha}$.

Next we perform a preliminary analysis of the bifurcation.

Lemma 2.1. Any solution $(\lambda, u)$ to (1.3) near the bifurcation point $\left(\lambda_{2}, 0\right)$ has the form

$$
\left\{\begin{array}{l}
\lambda=\lambda_{2}+s^{2} \sigma+o\left(s^{2}\right), \\
u=s \phi_{(\alpha+o(1))}+O\left(s^{3}\right),
\end{array}\right.
$$

with $\sigma$ given by

$$
\sigma=-\frac{\int_{\Omega} \phi_{\alpha}^{4}}{\int_{\Omega} \phi_{\alpha}^{2}}
$$

and $\alpha=0, \pi / 4, \pi / 2$ or $3 \pi / 4$.

Proof. Let $\left(\lambda_{n}, u_{n}\right)$ be a sequence of solutions to (1.3) such that $\lambda_{n} \rightarrow \lambda_{2}$ and $u_{n} \rightarrow 0$ in $C(\bar{\Omega})$. We make the following normalization:

$$
\hat{u}_{n}=\frac{u_{n}}{\left|u_{n}\right|_{\infty}} .
$$

Then $\hat{u}_{n}$ verifies the equation

$$
\left\{\begin{array}{cc}
\Delta \hat{u}_{n}+\lambda_{n} \hat{u}_{n}+\hat{u}_{n} u_{n}^{2}\left(1+\theta\left(x, u_{n}\right)\right)=0 & \text { in } \Omega, \\
\hat{u}_{n}=0 & \text { on } \partial \Omega .
\end{array}\right.
$$

Thus, taking into account that $\left|\hat{u}_{n}\right|_{\infty}=1$ and that (2.3) is equivalent to a fixed point equation for a compact operator in $C(\bar{\Omega})$, we have, passing to a subsequence (still denoted by $\hat{u}_{n}$ ) that $\hat{u}_{n} \rightarrow u_{0}$ in $C(\bar{\Omega})$, with $\left|u_{0}\right|_{\infty}=1$, and

$$
\left\{\begin{array}{ccc}
\Delta u_{0}+\lambda_{2} u_{0}=0 & \text { in } \quad \Omega, \\
u_{0}=0 & \text { on } \partial \Omega .
\end{array}\right.
$$

It follows that $u_{0}=c \phi_{\alpha}$ for some $\alpha \in[0,2 \pi)$ and $c=\left|\phi_{\alpha}\right|_{\infty}^{-1} \neq 0$. Writing $\hat{u}_{n}=\phi_{n}+\psi_{n}$, with $\phi_{n} \in M, \psi_{n} \in M^{\perp}$ (so that $\phi_{n} \rightarrow c \phi_{\alpha}$ and $\psi_{n} \rightarrow 0$ ) and $t_{n}=\left|u_{n}\right|_{\infty}$, and simplifying in (2.3), we arrive at

$$
\begin{gathered}
\Delta \psi_{n}+\lambda_{2} \psi_{n}+\left(\lambda_{n}-\lambda_{2}\right)\left(\phi_{n}+\psi_{n}\right) \\
+t_{n}^{2}\left(\phi_{n}+\psi_{n}\right)^{3}\left(1+\theta\left(x, t_{n}\left(\phi_{n}+\psi_{n}\right)\right)\right)=0 \text { in } \Omega .
\end{gathered}
$$

Multiplying by $\phi_{\alpha}$ and integrating by parts we obtain

$$
\frac{\lambda_{n}-\lambda_{2}}{t_{n}^{2}} \int_{\Omega} \phi_{n} \phi_{\alpha}+\int_{\Omega}\left(\phi_{n}+\psi_{n}\right)^{3}\left(1+\theta\left(x, t_{n}\left(\phi_{n}+\psi_{n}\right)\right) \phi_{\alpha}=0,\right.
$$

from which it follows after passing to the limit that

$$
\lim _{n \rightarrow+\infty} \frac{\lambda_{n}-\lambda_{2}}{t_{n}^{2}}=-c^{2} \frac{\int_{\Omega} \phi_{\alpha}^{4}}{\int_{\Omega} \phi_{\alpha}^{2}} .
$$

Similarly, multiplying (2.4) by $\psi_{\alpha}$, integrating by parts and passing to the limit we conclude

$$
\int_{\Omega} \phi_{\alpha}^{3} \psi_{\alpha}=0 .
$$


An important conclusion is obtained from this condition. It can be checked that

$$
\int_{0}^{\pi} \int_{0}^{\pi} \phi_{\alpha}(x, y)^{3} \psi_{\alpha}(x, y) d x d y=-\frac{3}{128} \pi^{2} \sin 2 \alpha \cos 2 \alpha,
$$

so that the bifurcation is only possible from four values of $\alpha$, namely $\alpha=0, \pi / 4, \pi / 2$, $3 \pi / 2$ (observe that there are other values of $\alpha$ leading to the same eigenfunctions with a change of sign, since $\phi_{\pi+\alpha}=-\phi_{\alpha}$ ).

Finally notice that it follows from (2.4) that $\Delta \psi_{n}+\lambda_{2} \psi_{n}=O\left(t_{n}^{2}\right)$. Since $\left(\Delta+\lambda_{2}\right)^{-1}$ is a bounded linear operator from $M^{\perp}$ into itself, we also have that $\psi_{n}=O\left(t_{n}^{2}\right)$. As a consequence of this analysis, and after a rescaling in the parameter $s$, the result of the lemma follows.

Now we turn to the actual construction of the bifurcated branches. Let $\alpha_{0}$ be fixed as one of the four values $0, \pi / 4, \pi / 2,3 \pi / 4$ given above, and $\sigma_{0}$ be the corresponding value given by (2.2). For $s$ small we want to solve

$$
\left\{\begin{array}{cc}
\Delta \psi+\lambda_{2} \psi+\sigma \phi_{\alpha}+s^{2} \sigma \psi+\left(\phi_{\alpha}+s^{2} \psi\right)^{3}\left(1+\theta\left(x, s \phi_{\alpha}+s^{3} \psi\right)\right)=0 & \text { in } \quad \Omega, \\
\psi=0 & \text { on } \quad \partial \Omega,
\end{array}\right.
$$

with $\psi \in M^{\perp}$. Denoting by $K$ the inverse of $\Delta$ (which is a compact, linear operator from $C(\bar{\Omega})$ into itself), this is equivalent to solving $H(\alpha, \sigma, \psi, s)=0$, for $\alpha \sim \alpha_{0}$, $\sigma \sim \sigma_{0}, \psi \in M^{\perp}, \psi \sim \psi_{0}$, where

$$
H(\alpha, \sigma, \psi, s)=\psi+K\left(\lambda_{2} \psi+\sigma \phi_{\alpha}+s^{2} \sigma \psi+\left(\phi_{\alpha}+s^{2} \psi\right)^{3}\left(1+\theta\left(x, s \phi_{\alpha}+s^{3} \psi\right)\right)\right),
$$

and $\psi_{0} \in M^{\perp}$ is the unique solution of

$$
\left\{\begin{array}{ccc}
\Delta \psi+\lambda_{2} \psi+\sigma_{0} \phi_{\alpha_{0}}+\phi_{\alpha_{0}}^{3}=0 & \text { in } & \Omega \\
\psi=0 & \text { on } & \partial \Omega
\end{array} .\right.
$$

Let us see that the implicit function theorem can be applied in our setting. Notice that $H$ is a $C^{1}$ function of its arguments in a neighbourhood of $\left(\alpha_{0}, \sigma_{0}, \psi_{0}, 0\right)$ in $\mathbb{R} \times \mathbb{R} \times M^{\perp} \times \mathbb{R}$. Also, $H\left(\alpha_{0}, \sigma_{0}, \psi_{0}, 0\right)=0$, and

$$
D H_{(\alpha, \sigma, \psi)}\left(\alpha_{0}, \sigma_{0}, \psi_{0}, 0\right)(\hat{\alpha}, \hat{\sigma}, \hat{\psi})=\hat{\psi}+K\left(\lambda_{2} \hat{\psi}+\hat{\sigma} \phi_{\alpha_{0}}-\hat{\alpha}\left(\sigma_{0} \psi_{\alpha_{0}}+3 \phi_{\alpha_{0}}^{2} \psi_{\alpha_{0}}\right)\right) \text {. }
$$

Assume that $D H_{(\alpha, \sigma, \psi)}\left(\alpha_{0}, \sigma_{0}, \psi_{0}, 0\right)(\hat{\alpha}, \hat{\sigma}, \hat{\psi})=0$ for some $(\hat{\alpha}, \hat{\sigma}, \hat{\psi}) \in \mathbb{R} \times \mathbb{R} \times M^{\perp}$. This means that $\hat{\psi}$ solves the problem

$$
\left\{\begin{array}{ccc}
\Delta \hat{\psi}+\lambda_{2} \hat{\psi}+\hat{\sigma} \phi_{\alpha_{0}}-\left(\sigma_{0} \psi_{\alpha_{0}}+3 \phi_{\alpha_{0}}^{2} \psi_{\alpha_{0}}\right) \hat{\alpha}=0 & \text { in } \quad \Omega, \\
\hat{\psi}=0 & \text { on } \quad \partial \Omega .
\end{array}\right.
$$

Multiplying by $\phi_{\alpha_{0}}$, integrating in $\Omega$ and performing an integration by parts, we arrive at $\hat{\sigma}=0$ since (2.5) holds. Multiplying by $\psi_{\alpha_{0}}$ instead, we get

$$
\hat{\alpha}\left(\sigma_{0} \int_{\Omega} \psi_{\alpha_{0}}^{2}+3 \int_{\Omega} \phi_{\alpha_{0}}^{2} \psi_{\alpha_{0}}^{2}\right)=0 .
$$

It is easy to see that the term inside brackets is always nonzero . Thus $\hat{\alpha}=0$, and (2.6) leads to $\hat{\psi}=0$. To summarize, $D H_{(\alpha, \sigma, \psi)}\left(\alpha_{0}, \sigma_{0}, \psi_{0}, 0\right)$ is one-to-one and hence an isomorphism, since it can be identified with a compact perturbation of the identity.

The implicit function theorem applies to give $\varepsilon>0$ and three $C^{1}$ functions $\alpha:(-\varepsilon, \varepsilon) \rightarrow \mathbb{R}, \sigma:(-\varepsilon, \varepsilon) \rightarrow \mathbb{R}$ and $\psi:(-\varepsilon, \varepsilon) \rightarrow M^{\perp}$ such that $\alpha(0)=$ $\alpha_{0}, \sigma(0)=\sigma_{0}, \psi(0)=\psi_{0}$ and the set of solutions of $H(\alpha, \sigma, \psi, s)=0$ near the point $\left(\alpha_{0}, \sigma_{0}, \psi_{0}, 0\right)$ reduces to $(\alpha(s), \sigma(s), \psi(s), s)$. This conclusion (together with 
Lemma 2.1) gives in particular a unique curve of solutions to (1.3) such that $u(s) \sim$ $s \phi_{\alpha_{0}}$ as $s \rightarrow 0$. Since $\alpha_{0}$ can be taken as any of the four values $0, \pi / 4, \pi / 2,3 \pi / 4$, we have exactly four branches of solutions near the bifurcation point $\left(\lambda_{2}, 0\right)$. The proof of (1.6) follows from (2.2) and a straightforward calculation. The first part of the theorem is thus complete.

\section{Computation of Morse indices}

In this section we compute the Morse index of the solutions corresponding to the four bifurcating branches found in the previous section. Thus we need to find the number of negative eigenvalues $\mu$ of the problem

$$
\left\{\begin{array}{ccc}
\Delta \phi+\lambda \phi+u^{2}\left(3(1+\theta(x, u))+u \theta_{u}(x, u)\right) \phi+\mu \phi=0 & \text { in } \quad \Omega, \\
\phi=0 & \text { on } \partial \Omega .
\end{array}\right.
$$

Setting $\lambda=\lambda_{2}+s^{2} \sigma, u=s \phi_{\alpha}+s^{3} \psi$, and simplifying, we obtain

$$
\left\{\begin{array}{l}
\Delta \phi+\lambda_{2} \phi+s^{2} \sigma \phi+3 s^{2}\left(\phi_{\alpha}+s^{2} \psi\right)^{2}\left(1+\theta\left(x, s \phi_{\alpha}+s^{3} \psi\right)\right) \\
\quad+s^{2}\left(\phi_{\alpha}+s^{2} \psi\right)^{3} \theta_{u}\left(x, s \phi_{\alpha}+s^{3} \psi\right) \phi+\mu \phi=0 \text { in } \Omega, \\
\phi=0 \text { on } \partial \Omega,
\end{array}\right.
$$

where now $\mu=\mu(s), \phi=\phi(s)$. We normalize $\phi$ by $|\phi|_{L^{2}}^{2}=\pi / 2$. As $s$ goes to zero, it follows that $\mu(s)$ and $\phi(s)$ converge respectively to an eigenvalue and eigenfuncion of the problem

$$
\left\{\begin{array}{ccc}
\Delta \phi+\lambda_{2} \phi+\mu \phi=0 & \text { in } & \Omega, \\
\phi=0 & \text { on } & \partial \Omega
\end{array}\right.
$$

whose set of eigenvalues is $\left\{\lambda_{1}-\lambda_{2}, 0, \lambda_{3}-\lambda_{2}, \ldots\right\}$. Thus, for $s$ small the first eigenvalue of (3.1) is always negative and close to $\lambda_{1}-\lambda_{2}$. There is also one positive eigenvalue close to $\lambda_{3}-\lambda_{2}$, and we have to decide what happens with the eigenvalues close to 0 . Notice that for $s$ small there are always two such eigenvalues $\mu_{1}(s), \mu_{2}(s)$ (counting multiplicity), since the proper subspace has to be two-dimensional.

Let $\phi_{1}(s), \phi_{2}(s)$ denote the orthogonal eigenfunctions corresponding to these two eigenvalues. Then $\phi_{i}(s) \rightarrow A_{i} \phi_{\alpha}+B_{i} \psi_{\alpha}$ as $s \rightarrow 0$, where $A_{i}^{2}+B_{i}^{2}=1$ and $A_{1} A_{2}+B_{1} B_{2}=0$. Multiplying (3.1) by $\phi_{\alpha}$, integrating over $\Omega$, and passing to the limit as $s \rightarrow 0$, we have that

$$
A_{i}\left(\sigma \int_{\Omega} \phi_{\alpha}^{2}+3 \int_{\Omega} \phi_{\alpha}^{4}+\lim _{s \rightarrow 0} \frac{\mu_{i}(s)}{s^{2}} \int_{\Omega} \phi_{\alpha}^{2}\right)=0,
$$

and similarly, testing the equation against $\psi_{\alpha}$,

$$
B_{i}\left(\sigma \int_{\Omega} \psi_{\alpha}^{2}+3 \int_{\Omega} \phi_{\alpha}^{2} \psi_{\alpha}^{2}+\lim _{s \rightarrow 0} \frac{\mu_{i}(s)}{s^{2}} \int_{\Omega} \psi_{\alpha}^{2}\right)=0 .
$$

Now we have to distinguish between branches. If $\alpha=0$ or $\pi / 2$, these equalities read

$$
\begin{aligned}
& A_{i}\left(\lim _{s \rightarrow 0} \frac{\mu_{i}(s)}{s^{2}}+\frac{9}{8}\right)=0, \\
& B_{i}\left(\lim _{s \rightarrow 0} \frac{\mu_{i}(s)}{s^{2}}+\frac{3}{16}\right)=0 .
\end{aligned}
$$

Thus we obtain that, say, $A_{i}=0$ or $B_{i}=0$ for both $i=1,2$; hence for $s$ small both eigenvalues are negative. 
If, on the contrary, $\alpha=\pi / 4$ or $3 \pi / 4$, (3.2) becomes

$$
\begin{aligned}
& A_{i}\left(\lim _{s \rightarrow 0} \frac{\mu_{i}(s)}{s^{2}}+\frac{21}{16}\right)=0, \\
& B_{i}\left(\lim _{s \rightarrow 0} \frac{\mu_{i}(s)}{s^{2}}-\frac{3}{16}\right)=0 .
\end{aligned}
$$

Arguing as before, this shows that there is a positive eigenvalue and a negative one. To summarize, the first two branches of the bifurcation diagram, corresponding to $\alpha=0, \frac{\pi}{2}$, have Morse index three, while the latter ones, corresponding to $\alpha=$ $\frac{\pi}{4}, \frac{3 \pi}{4}$, have Morse index two.

This concludes the proof of Theorem 1.1.

\section{ACKNOWLEDGEMENTS}

The first author has been supported by Fondecyt Lineas Complementarias grant 8000010, and FONDAP Matemáticas Aplicadas, Chile. The second author has been supported by grant MCYT \# BFM2001-3894 (Spain). The third author has been supported by Progetto Nazionale ex $40 \%$ "Metodi variazionali e topologici nello studio di fenomeni non lineari", Italy.

\section{REFERENCES}

1. Ambrosetti A. And Prodi G., A primer of Nonlinear Analysis, Cambridge Studies in Advanced Mathematics, Cambridge University Press (1993). MR 94f:58016

2. Böнme R., Die Lösung der Verzweigungsgleichungen für nichtlineare Eigenwertprobleme, Math. Z. 127 (1972), 105-126. MR 47:910

3. Crandall M.G. and Rabinowitz P.H., Bifurcation from simple eigenvalues, J. Funct. Anal. 8 (1971), 321-340. MR 44:5836

4. Krasnoselski M.A., Topological methods in the theory of nonlinear integral equations, MacMillan, New York (1964). MR 28:2414

5. Marino A., La biforcazione nel caso variazionale, Confer. Sem. Mat. Univ. Bari (132) (1973), 14pp. MR 50:1068

6. Rabinowitz P.H., A bifurcation theorem for potential operators, J. Funct. Anal. 25 (4) (1977), 412-424. MR 57:3928

Departamento de Ingeniería Matemática and CMM, Universidad de Chile, Casilla 170 Correo 3, Santiago, Chile

E-mail address: delpino@dim.uchile.cl

Departamento de Análisis Matemático, Universidad de La Laguna, C/ Astrofísico FCo. SÁnchez S/N - 38271 La Laguna, Spain

E-mail address: jjgarmel@ull.es

Dipartimento di Matematica, Politecnico di Torino, Corso Duca degli Abruzzi, 24 10129 TORINO, ITALY

E-mail address: musso@calvino.polito.it 